For Internal Distribution Only

Accelerator Division

Alternating Gradient Synchrotron Department

BROOKHAVEN NATIONAL LABORATORY

Upton, New York 11973

Accelerator Division

Technical Note

AGS/AD/Tech. Note No. 430

THE PARAMETERS OF THE BARE AGS

E. J. Bleser

March 15, 1996 


\title{
THE PARAMETERS OF THE BARE AGS
}

\author{
E. J. Bleser
}

\section{Summary}

This note briefly recapitulates the properties of the bare AGS as calculated by MAD using a revised set of input parameters. It slightly changes the previously standard tune values and offers a parameterization of the tunes and chromaticities as functions of the momentum.

\section{Modeling and Calculations}

Technical Note No. 429 parameterized the quadrupole and sextupole fields of the main AGS magnets as functions of momentum. This note uses these parameterizations in MAD to calculate the tune and chromaticity of the AGS. It then parameterizes these results as a function of momentum, resulting in a set of simple formulas which give the tune and chromaticity for any value of the momentum of the AGS. The contributions of this note are 1.) the formulas just discussed, and 2.) new calculated values for the tunes, presumably better than what we have been using. This change in the calculated tunes is discussed in Appendix I.

These calculations are for the "Bare AGS", that is for the AGS with just the main magnets powered. These calculations are also for the "DC AGS", that is for the case where the magnetic field is not changing and there are no eddy currents. They provide a baseline set of numbers which can be adjusted to take account of low field and high field correction magnet systems and which can be adjusted for eddy current effects. The accuracy of these calculations should be about one part in a thousand. Careful tune measurements may have an accuracy better than this and experiment may dictate modifying the results presented here.

The file used to input the AGS to MAD has been greatly elaborated to make modeling the AGS easier for the user. It now includes the standard geometry as before, all the high field correction systems, and parameterized momentum and current inputs. The user must simply specify his operating momentum and the currents at which he wants to operate his correction systems. Running MAD is somewhat analogous to operating the AGS. 


\section{Tune and Chromaticity Results}

Table 1 gives the horizontal and vertical tunes, $\mathrm{Q}_{\mathrm{x}}$ and $\mathrm{Q}_{\mathrm{y}}$, the normalized horizontal and vertical chromaticities, $Q_{x}^{\prime} / Q_{x}$ and $Q^{\prime} / Q_{y}$, and the mean of the chromaticities. These values were calculated by MAD for input values of the momentum, $\mathrm{P}$, from 1 to $32 \mathrm{GeV} / \mathrm{c}$. Also given in the table are the values of the main magnet current, I, calculated from the formula in Tech Note No. 424. The tune results are plotted in Figures 1, 2, and 3, the chromaticity results in Figure 4.

Note that in Figures 1 and 2 the tune droops significantly at high field due to the saturation of the AGS magnets. The curves drawn in these figures are calculated from the formulas given in Table 2, resulting from a fit over the range from 1 to $32 \mathrm{GeV} / \mathrm{c}$. Figure 3 shows the tunes over our operating range. Around $2 \mathrm{GeV} / \mathrm{c}$ the fit is not perfect but it is within our measurement accuracy. As discussed in Appendix I, the old results for $\mathrm{Q}_{\mathrm{y}}$ were around 8.75 rather than 8.76 as calculated now.

Figure 4 shows that the mean of the normalized horizontal and vertical chromaticities is close to -1 , as we should expect for a nearly round accelerator. The sextupole component in the main magnets splits the horizontal and vertical chromaticities. At high fields a large saturation sextupole component increases this splitting. Below $20 \mathrm{GeV} / \mathrm{c}$ eddy currents in the vacuum chamber produce an additional large sextupole field which will make any nonDC measurements differ significantly from these calculations. Table 3 contains the parameters for the curves fitted to these data.

The fitted results must be checked against experiment, but in principle the is no further need to run "bare AGS" tune calculations in MAD.

\section{Twiss Results}

In Tech Note No. 297 Auerbach presented extensive results for the Twiss parameters. The present calculations may be slightly different as discussed in the Appendix, but not significantly so. This note presents a calculation at $15 \mathrm{GeV} / \mathrm{c}$. Table 4 lists the various parameters for one superperiod, A, which starts at the entrance to magnet A1. The high field correction magnets are specified in the element column but are not powered. In other superperiods the correction magnets may be different, but as long as they are not powered, all the superperiod Twiss parameters will be identical to the results given here. These results are plotted in Figures 5, 6, and 7. 


\section{APPENDIX I. COMPARISON OF THE OLD AND NEW CALCULATIONS}

Until the present calculations, the quadrupole gradients used in MAD assumed that the gradients in the open magnets were the same for both the long, A, magnets and the short, $B$, magnets. In actuality since the end fields are a larger effect in the short magnets than in the long magnets, the gradient in B should be slightly lower than the gradient in A. Detailed integrations by Thern have confirmed this assumption and his more correct values have been used for this note and installed in MAD. Using the old gradients reproduces the earlier calculations, using the new gradients reduces the calculated tunes by about 0.01 units as shown in Figure A1. 
TABLE 1

THE BARE AGS

WITH NO EDDY CURRENTS

\begin{tabular}{|c|c|c|c|c|c|c|}
\hline $\mathbf{P}$ & 1 & \multirow[t]{2}{*}{$Q \mathbf{Q x}$} & \multirow[t]{2}{*}{$\overline{Q y}$} & $Q x^{\prime} / Q x$ & Qy'/Qy & MEAN of \\
\hline GeV/c & Amperes & & & $m^{-1}$ & $m^{-1}$ & $Q x^{\prime} / Q x \& Q y^{\prime} / Q y$ \\
\hline 1 & 169.4 & 8.706 & 8.758 & -2.466 & 0.250 & -1.108 \\
\hline 2 & 343.5 & 8.711 & 8.764 & -2.400 & 0.187 & -1.106 \\
\hline 3 & 516.5 & $\overline{8.711}$ & 8.765 & -2.368 & 0.159 & -1.105 \\
\hline 4 & 689.4 & 8.710 & 8.764 & -2.343 & 0.136 & -1.103 \\
\hline 5 & 862.1 & 8.708 & 8.763 & -2.320 & 0.116 & -1.102 \\
\hline 6 & 1034.7 & 8.707 & 8.762 & -2.301 & 0.099 & -1.101 \\
\hline 7 & 1207.3 & 8.706 & 8.761 & -2.287 & 0.085 & -1.101 \\
\hline 8 & 1379.7 & 8.706 & 8.760 & -2.277 & 0.076 & -1.100 \\
\hline 9 & 1552.0 & 8.706 & 8.760 & -2.273 & 0.072 & -1.100 \\
\hline 10 & 1724.2 & 8.706 & 8.760 & -2.274 & 0.073 & -1.101 \\
\hline 11 & 1896.5 & 8.706 & 8.760 & -2.280 & 0.077 & -1.101 \\
\hline 12 & 2068.8 & 8.706 & 8.761 & -2.289 & 0.086 & -1.102 \\
\hline 13 & 2241.3 & 8.706 & 8.761 & -2.302 & 0.097 & -1.103 \\
\hline$\overline{14}$ & 2414.0 & 8.706 & 8.760 & -2.317 & 0.111 & -1.103 \\
\hline 15 & 2587.0 & 8.705 & 8.760 & -2.333 & 0.126 & -1.104 \\
\hline 16 & 2760.4 & 8.705 & 8.759 & -2.350 & 0.141 & -1.104 \\
\hline 17 & 2934.1 & 8.703 & 8.757 & -2.368 & 0.158 & -1.105 \\
\hline 18 & 3108.1 & 8.702 & 8.755 & -2.388 & 0.177 & -1.105 \\
\hline 19 & 3282.4 & 8.700 & 8.753 & -2.410 & 0.198 & -1.106 \\
\hline 20 & 3456.9 & 8.698 & 8.750 & -2.438 & 0.225 & -1.107 \\
\hline 21 & 3631.7 & 8.696 & 8.747 & -2.476 & 0.261 & -1.108 \\
\hline 22 & 3806.7 & 8.693 & 8.743 & -2.527 & 0.309 & -1.109 \\
\hline 23 & 3982.2 & 8.690 & 8.738 & -2.600 & 0.377 & -1.111 \\
\hline 24 & 4158.7 & 8.686 & 8.733 & -2.703 & 0.473 & -1.115 \\
\hline 25 & 4336.8 & 8.680 & 8.725 & -2.847 & 0.606 & -1.120 \\
\hline 26 & 4517.7 & 8.672 & 8.716 & -3.046 & 0.790 & -1.128 \\
\hline 27 & 4703.2 & 8.661 & 8.703 & -3.317 & 1.040 & -1.139 \\
\hline 28 & 4895.6 & 8.645 & 8.686 & -3.683 & 1.376 & -1.153 \\
\hline 29 & 5098.3 & 8.622 & 8.662 & -4.170 & 1.824 & -1.173 \\
\hline 30 & 5315.8 & 8.589 & 8.629 & -4.814 & 2.416 & -1.199 \\
\hline 31 & 5553.9 & 8.545 & 8.585 & -5.663 & 3.197 & -1.233 \\
\hline 32 & 5819.9 & 8.484 & 8.527 & -6.784 & 4.228 & -1.278 \\
\hline
\end{tabular}


TABLE 2

THE TUNE of the BARE AGS

as a FUNCTION of the MOMENTUM

in $\mathrm{GeV} / \mathrm{c}$

$$
Q=q_{0}+q_{1} P+q_{2} P^{2}+q_{3} P^{3}+q_{4} P^{4}+q_{5} P^{5}+q_{6} P^{6}
$$

\begin{tabular}{|l|r|r|}
\cline { 2 - 3 } \multicolumn{1}{c|}{} & \multicolumn{1}{c|}{$\boldsymbol{Q}_{x}$} & \multicolumn{1}{c|}{$\boldsymbol{Q}_{\boldsymbol{y}}$} \\
\hline $\boldsymbol{q}_{0}$ & 8.7005 & 8.7497 \\
\hline $\boldsymbol{q}_{\boldsymbol{1}}$ & $9.0027 \mathrm{E}-03$ & $1.1705 \mathrm{E}-02$ \\
\hline $\boldsymbol{q}_{\mathbf{2}}$ & $-2.7937 \mathrm{E}-03$ & $-3.2926 \mathrm{E}-03$ \\
\hline $\boldsymbol{q}_{3}$ & $3.6114 \mathrm{E}-04$ & $4.0215 \mathrm{E}-04$ \\
\hline $\boldsymbol{q}_{4}$ & $-2.2733 \mathrm{E}-05$ & $-2.4222 \mathrm{E}-05$ \\
\hline $\boldsymbol{q}_{5}$ & $6.8948 \mathrm{E}-07$ & $7.0669 \mathrm{E}-07$ \\
\hline $\boldsymbol{q}_{\boldsymbol{6}}$ & $-8.1737 \mathrm{E}-09$ & $-8.1188 \mathrm{E}-09$ \\
\hline
\end{tabular}

\section{TABLE 3}

THE CHROMATICITY of the BARE AGS as a FUNCTION of the MOMENTUM in $\mathrm{GeV} / \mathrm{c}$

$$
Q^{\prime} Q=r_{0}+r_{1} P+r_{2} P^{2}+r_{3} P^{3}+r_{4} P^{4}+r_{5} P^{5}+r_{6} P^{6}
$$

\begin{tabular}{|l|r|r|}
\cline { 2 - 3 } \multicolumn{1}{c|}{} & \multicolumn{1}{c|}{$Q^{\prime}{ }_{x} / Q_{x}$} & \multicolumn{1}{c|}{$Q^{\prime}{ }_{y} / Q_{y}$} \\
\hline$r_{0}$ & -2.5374 & 0.3159 \\
\hline$r_{1}$ & $8.4661 \mathrm{E}-02$ & $-8.1296 \mathrm{E}-02$ \\
\hline$r_{2}$ & $-1.3439 \mathrm{E}-02$ & $1.3069 \mathrm{E}-03$ \\
\hline$r_{3}$ & $1.4142 \mathrm{E}-03$ & $-1.4019 \mathrm{E}-03$ \\
\hline$r_{4}$ & $-9.8939 \mathrm{E}-05$ & $9.7259 \mathrm{E}-05$ \\
\hline$r_{5}$ & $3.6948 \mathrm{E}-06$ & $-3.5710 \mathrm{E}-06$ \\
\hline$r_{6}$ & $-5.5707 \mathrm{E}-08$ & $5.2981 \mathrm{E}-08$ \\
\hline
\end{tabular}


TABLE 4. SUPERPERIOD A OF THE AGS

\begin{tabular}{|c|c|c|c|c|c|c|c|c|c|c|c|}
\hline & \multirow{2}{*}{$\begin{array}{l}\text { ELE- } \\
\text { MENT }\end{array}$} & \multirow{2}{*}{$\begin{array}{l}\text { MAGNET } \\
\text { TYPE }\end{array}$} & $\mathbf{S}$ & BETAX & ALFAX & MUX & $\overline{D X}$ & DPX & BETAY & ALFAY & MUY \\
\hline & & & meters & meters & & $2 \mathrm{PI}$ & meters & & meters & & $2 \mathrm{PI}$ \\
\hline 0 & BEGIN SUP & IPER A & 0 & 19.753 & -1.569 & 0 & 2.054 & 0.148 & 11.741 & 1.028 & 0 \\
\hline 1 & $\mathrm{~A} 1$ & $\mathrm{BF}$ & 2.007 & 22.305 & 0.381 & 0.015 & 2.167 & -0.037 & 10.251 & -0.238 & 0.03 \\
\hline 2 & & & 2.616 & 21.86 & 0.35 & 0.019 & 2.144 & -0.037 & 10.579 & -0.301 & 0.04 \\
\hline 3 & $\overline{A 2}$ & $\mathrm{BF}$ & 4.623 & 16.824 & 1.994 & 0.035 & 1.888 & -0.213 & 14.591 & -1.827 & 0.066 \\
\hline 4 & & & 4.91 & 15.704 & 1.909 & 0.038 & 1.827 & -0.213 & 15.664 & -1.912 & 0.069 \\
\hline 5 & \begin{tabular}{|l|} 
PUE $\mathrm{A02}$ \\
\end{tabular} & & 4.91 & 15.704 & 1.909 & 0.038 & 1.827 & -0.213 & 15.664 & -1.912 & 0.069 \\
\hline 6 & & & 5.232 & 14.503 & 1.814 & 0.041 & 1.758 & -0.213 & 16.928 & -2.008 & 0.072 \\
\hline 7 & $\overline{A 3}$ & $C D$ & 7.62 & 10.395 & 0.063 & 0.074 & 1.508 & -0.001 & 22.11 & 0.042 & 0.091 \\
\hline 8 & & & 7.991 & 10.362 & 0.027 & 0.08 & 1.508 & -0.001 & 22.085 & 0.025 & 0.094 \\
\hline 9 & QHFV & & 8.382 & 10.356 & -0.011 & 0.086 & 1.507 & -0.001 & 22.073 & 0.008 & 0.097 \\
\hline 10 & QPOL & & 8.773 & 10.379 & -0.049 & 0.092 & 1.507 & -0.001 & 22.073 & -0.01 & 0.1 \\
\hline 11 & & & 9.144 & 10.429 & -0.085 & 0.097 & 1.507 & -0.001 & 22.087 & -0.027 & 0.102 \\
\hline 12 & A4 & $\overline{C D}$ & 11.531 & 14.673 & -1.854 & 0.13 & 1.751 & 0.211 & 16.851 & 2.013 & 0.121 \\
\hline 13 & & & 11.818 & 15.762 & -1.941 & 0.133 & 1.812 & 0.211 & 15.72 & 1.927 & 0.124 \\
\hline 14 & PUE A04 & & 11.818 & 15.762 & -1.941 & 0.133 & 1.812 & 0.211 & 15.72 & 1.927 & 0.124 \\
\hline 15 & & & 12.141 & 17.045 & -2.038 & 0.136 & 1.88 & 0.211 & 14.508 & 1.831 & 0.127 \\
\hline 16 & A5 & $\overline{A F}$ & 14.528 & 22.329 & 0.034 & 0.155 & 2.137 & 0 & 10.338 & 0.074 & 0.16 \\
\hline 17 & & & 15.29 & 22.303 & 0 & 0.16 & 2.137 & of & 10.282 & 0 & 0.172 \\
\hline 18 & & & 16.052 & 22.329 & -0.034 & 0.165 & 2.137 & 0 & 10.338 & -0.074 & 0.184 \\
\hline 19 & $\overline{A 6}$ & $\overline{A F}$ & 18.44 & 17.045 & 2.038 & 0.184 & 1.88 & -0.211 & 14.508 & -1.831 & 0.216 \\
\hline 20 & & & 19.049 & 14.673 & 1.854 & 0.19 & 1.751 & -0.211 & 16.851 & -2.013 & 0.222 \\
\hline 21 & A7 & $C D$ & 21.437 & 10.429 & 0.085 & 0.223 & 1.507 & 0.001 & 22.087 & 0.027 & 0.241 \\
\hline 22 & & & 21.871 & 10.373 & 0.043 & 0.229 & 1.507 & 0.001 & 22.072 & 0.007 & $\overline{0.244}$ \\
\hline 23 & SXV & & 22.526 & 10.359 & -0.021 & 0.239 & 1.508 & 0.001 & 22.082 & -0.022 & 0.249 \\
\hline 24 & & & 22.961 & 10.395 & -0.063 & 0.246 & 1.508 & 0.001 & 22.11 & -0.042 & 0.252 \\
\hline 25 & A8 & $\overline{C D}$ & 25.348 & 14.503 & -1.814 & 0.279 & 1.758 & 0.213 & 16.928 & 2.008 & 0.271 \\
\hline 26 & & & 25.635 & 15.569 & -1.899 & 0.282 & 1.819 & 0.213 & 15.8 & 1.923 & 0.274 \\
\hline 27 & PUE A08 & & 25.635 & 15.569 & -1.899 & 0.282 & 1.819 & 0.213 & 15.8 & 1.923 & 0.274 \\
\hline 28 & & & 25.958 & 16.824 & -1.994 & 0.285 & 1.888 & 0.213 & 14.591 & 1.827 & 0.277 \\
\hline 29 & A9 & $\overline{B F}$ & 27.964 & 21.86 & -0.35 & 0.301 & 2.144 & 0.037 & 10.579 & 0.301 & 0.304 \\
\hline 30 & & & 28.574 & 22.305 & -0.381 & 0.305 & 2.167 & 0.037 & 10.251 & 0.238 & 0.313 \\
\hline 31 & A10 & $\mathrm{BF}$ & 30.58 & 19.753 & 1.569 & 0.32 & 2.054 & -0.148 & 11.741 & -1.028 & 0.343 \\
\hline 32 & & & 32.104 & 15.377 & 1.302 & 0.334 & 1.829 & -0.148 & 15.282 & -1.295 & 0.362 \\
\hline
\end{tabular}


TABLE 4. continued

\begin{tabular}{|c|c|c|c|c|c|c|c|c|c|c|c|}
\hline \multirow{2}{*}{ 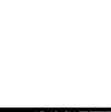 } & & \multirow{2}{*}{$\begin{array}{l}\text { MAGNET } \\
\text { TYPE }\end{array}$} & \multirow{2}{*}{$\frac{\mathbf{S}}{\text { meters }}$} & \multirow{2}{*}{\begin{tabular}{l|} 
BETAX \\
meters
\end{tabular}} & \multirow[t]{2}{*}{$\begin{array}{l}\text { ALFAX } \\
\end{array}$} & \multirow{2}{*}{$\frac{\mathrm{MUX}}{2 \mathrm{PI}}$} & \multirow{2}{*}{$\frac{\mathrm{DX}}{\text { meters }}$} & \multirow[t]{2}{*}{$\overline{D P X}$} & \multicolumn{2}{|c|}{\begin{tabular}{l|l|} 
BETAY & ALFAY \\
\end{tabular}} & \multirow{2}{*}{$\frac{\text { MUY }}{2 \mathrm{PI}}$} \\
\hline & & & & & & & & & meters & & \\
\hline 33 & \begin{tabular}{|l} 
ELE- \\
MENT \\
\end{tabular} & & 33.628 & 11.816 & 1.035 & 0.352 & 1.604 & \begin{tabular}{|c|c|}
-0.148 \\
\end{tabular} & 19.637 & -1.562 & 0.376 \\
\hline 34 & $\mathrm{~A} 11$ & $\mathrm{BD}$ & 35.635 & 10.315 & -0.239 & 0.382 & 1.481 & 0.023 & 22.18 & 0.379 & 0.39 \\
\hline 35 & & & 36.244 & 10.644 & -0.301 & 0.391 & 1.495 & 0.023 & 21.737 & 0.348 & 0.395 \\
\hline 36 & A12 & $\mathrm{BD}$ & 38.251 & 14.674 & -1.836 & 0.418 & 1.716 & 0.2 & 16.725 & 1.985 & 0.411 \\
\hline 37 & & & 38.538 & 15.753 & -1.922 & 0.421 & 1.773 & 0.2 & 15.611 & 1.9 & 0.414 \\
\hline 38 & \begin{tabular}{|l|} 
PUE A12 \\
\end{tabular} & & 38.538 & 15.753 & -1.922 & 0.421 & 1.773 & 0.2 & 15.611 & 1.9 & 0.414 \\
\hline 39 & & & 38.86 & 17.024 & -2.018 & 0.424 & 1.838 & 0.2 & 14.416 & 1.805 & 0.417 \\
\hline 40 & $\overline{\mathrm{A} 13}$ & $\mathrm{CF}$ & 41.248 & 22.231 & 0.042 & 0.443 & 2.078 & -0.004 & 10.325 & $\begin{array}{ll}0.063 \\
\end{array}$ & 0.45 \\
\hline 41 & & & 41.682 & 22.202 & 0.022 & 0.446 & 2.076 & -0.004 & 10.289 & 0.021 & 0.457 \\
\hline 42 & $\overline{S X H}$ & & 42.337 & 22.192 & -0.007 & 0.451 & 2.074 & -0.004 & 10.303 & \begin{tabular}{|c|}
-0.043 \\
\end{tabular} & 0.467 \\
\hline 43 & & & 42.772 & 22.207 & -0.027 & 0.454 & 2.072 & -0.004 & 10.358 & -0.085 & 0.474 \\
\hline 44 & A14 & $\overline{C F}$ & 45.159 & 16.945 & 2.024 & 0.472 & 1.816 & -0.206 & 14.582 & -1.844 & 0.506 \\
\hline 45 & & & 45.446 & 15.808 & 1.937 & 0.475 & 1.757 & -0.206 & 15.666 & -1.931 & 0.509 \\
\hline 46 & \begin{tabular}{|l|} 
PUE A14 \\
\end{tabular} & & 45.446 & 15.808 & 1.937 & 0.475 & 1.757 & -0.206 & 15.666 & -1.931 & 0.509 \\
\hline 47 & & & 45.769 & 14.59 & 1.84 & 0.479 & 1.691 & -0.206 & 16.942 & -2.028 & 0.513 \\
\hline 48 & A15 & $\overline{A D}$ & 48.156 & 10.402 & 0.074 & 0.511 & 1.451 & 0 & 22.199 & 0.034 & 0.531 \\
\hline 49 & & & 48.918 & 10.345 & of & 0.523 & 1.451 & 0 & 22.173 & 0 & 0.537 \\
\hline 50 & & & 49.68 & 10.402 & -0.074 & 0.534 & 1.451 & 0 & 22.199 & -0.034 & 0.542 \\
\hline 51 & A16 & $\overline{A D}$ & 52.068 & 14.59 & -1.84 & 0.567 & 1.691 & 0.206 & 16.942 & 2.028 & 0.561 \\
\hline 52 & & & 52.677 & 16.945 & -2.024 & 0.573 & 1.816 & 0.206 & 14.582 & 1.844 & 0.567 \\
\hline 53 & A17 & $\mathrm{CF}$ & 55.065 & 22.207 & 0.027 & 0.592 & 2.072 & 0.004 & 10.358 & 0.085 & 0.6 \\
\hline 54 & & & 55.436 & 22.193 & 0.01 & 0.595 & 2.074 & 0.004 & 10.309 & 0.049 & 0.605 \\
\hline 55 & QHFH & & 55.827 & 22.193 & -0.008 & 0.597 & 2.075 & 0.004 & 10.285 & 0.011 & 0.611 \\
\hline 56 & QGTR1 & & 56.218 & 22.205 & -0.025 & 0.6 & 2.077 & 0.004 & 10.292 & -0.027 & 0.618 \\
\hline 57 & & & 56.589 & 22.231 & -0.042 & 0.603 & 2.078 & 0.004 & 10.325 & -0.063 & 0.623 \\
\hline 58 & A18 & $\overline{C F}$ & 58.976 & 17.024 & 2.018 & 0.622 & 1.838 & -0.2 & 14.416 & -1.805 & 0.656 \\
\hline 59 & & & 59.263 & 15.89 & 1.933 & 0.624 & 1.78 & -0.2 & 15.476 & -1.889 & 0.659 \\
\hline 60 & \begin{tabular}{|l|} 
PUE A18 \\
\end{tabular} & & 59.263 & 15.89 & 1.933 & 0.624 & 1.78 & -0.2 & 15.476 & -1.889 & 0.659 \\
\hline 61 & & & 59.586 & 14.674 & 1.836 & 0.628 & 1.716 & -0.2 & 16.725 & -1.985 & 0.662 \\
\hline 62 & $\overline{A 19}$ & $\mathrm{BD}$ & 61.593 & 10.644 & 0.301 & 0.654 & 1.495 & -0.023 & 21.737 & -0.348 & 0.679 \\
\hline 63 & & & 62.202 & 10.315 & 0.239 & 0.663 & 1.481 & -0.023 & 22.18 & \begin{tabular}{|c|}
-0.379 \\
\end{tabular} & 0.683 \\
\hline 64 & $\overline{A 20}$ & $\mathrm{BD}$ & 64.209 & 11.816 & -1.035 & 0.693 & 1.604 & 0.148 & 19.637 & 1.562 & 0.698 \\
\hline 65 & & & 65.732 & 15.377 & -1.302 & 0.712 & 1.829 & 0.148 & 15.282 & 1.295 & 0.712 \\
\hline 66 & END SUPE & ERA & 67.256 & 19.753 & -1.569 & 0.725 & 2.054 & 0.148 & 11.741 & 1.028 & 0.73 \\
\hline
\end{tabular}


Qx vs $P$ for the BARE, DC AGS

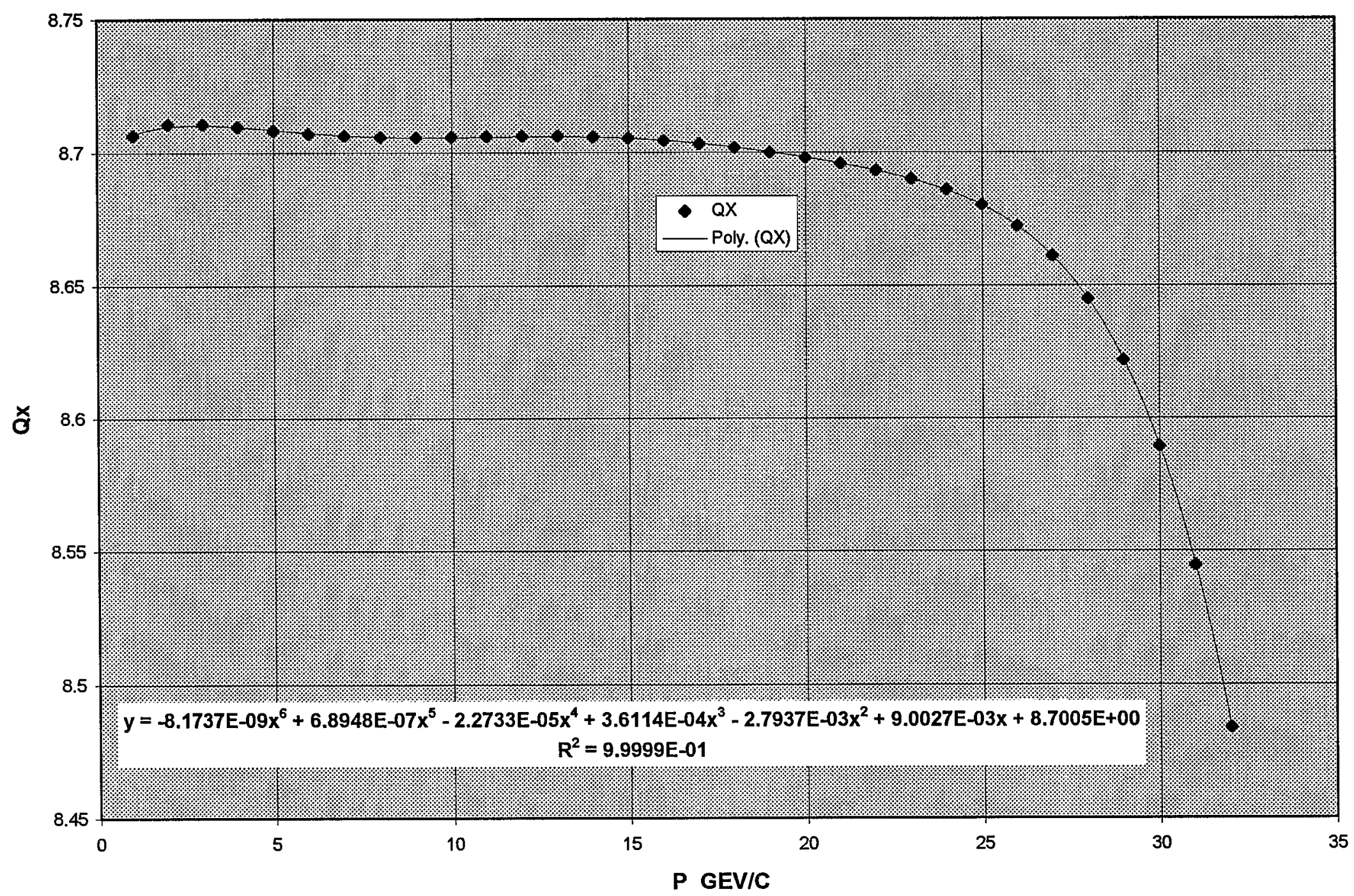


Qy vs $P$ for the BARE, DC AGS

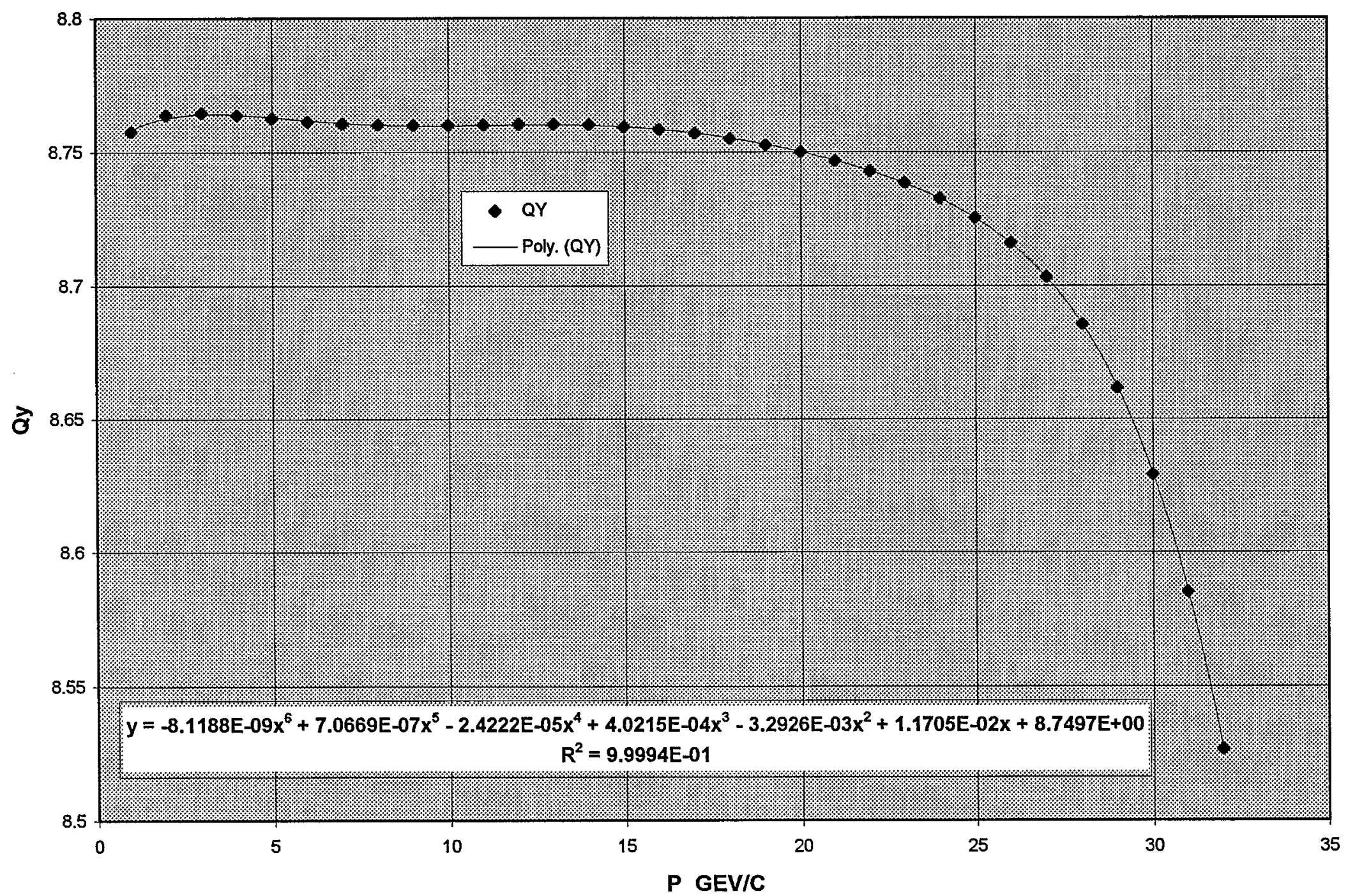


Qx \& Qy vs P for the BARE, DC AGS

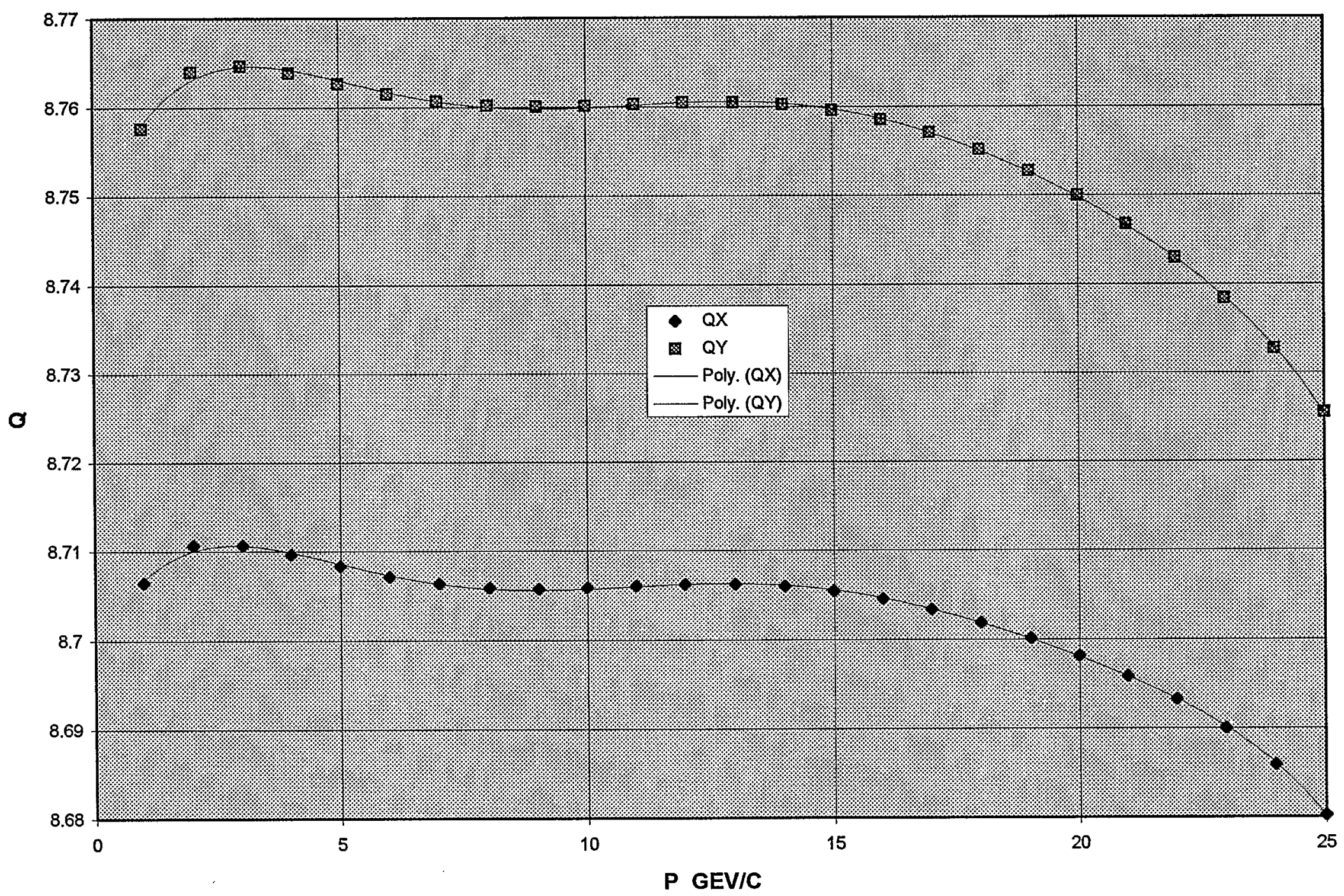


NORMALIZED CHROMATICITY vS P for THE BARE DC AGS

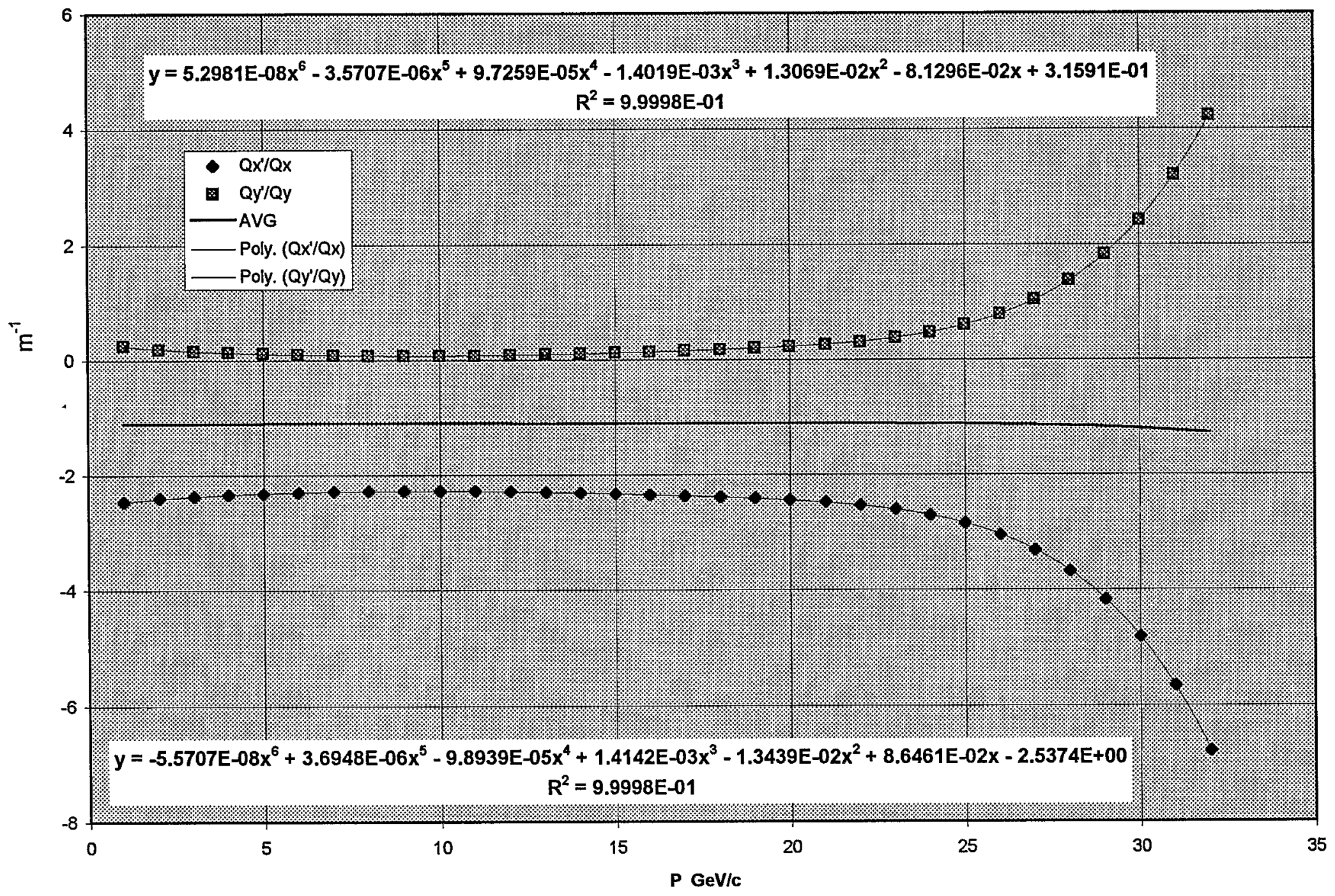


BETA X \& ALFA X vs S

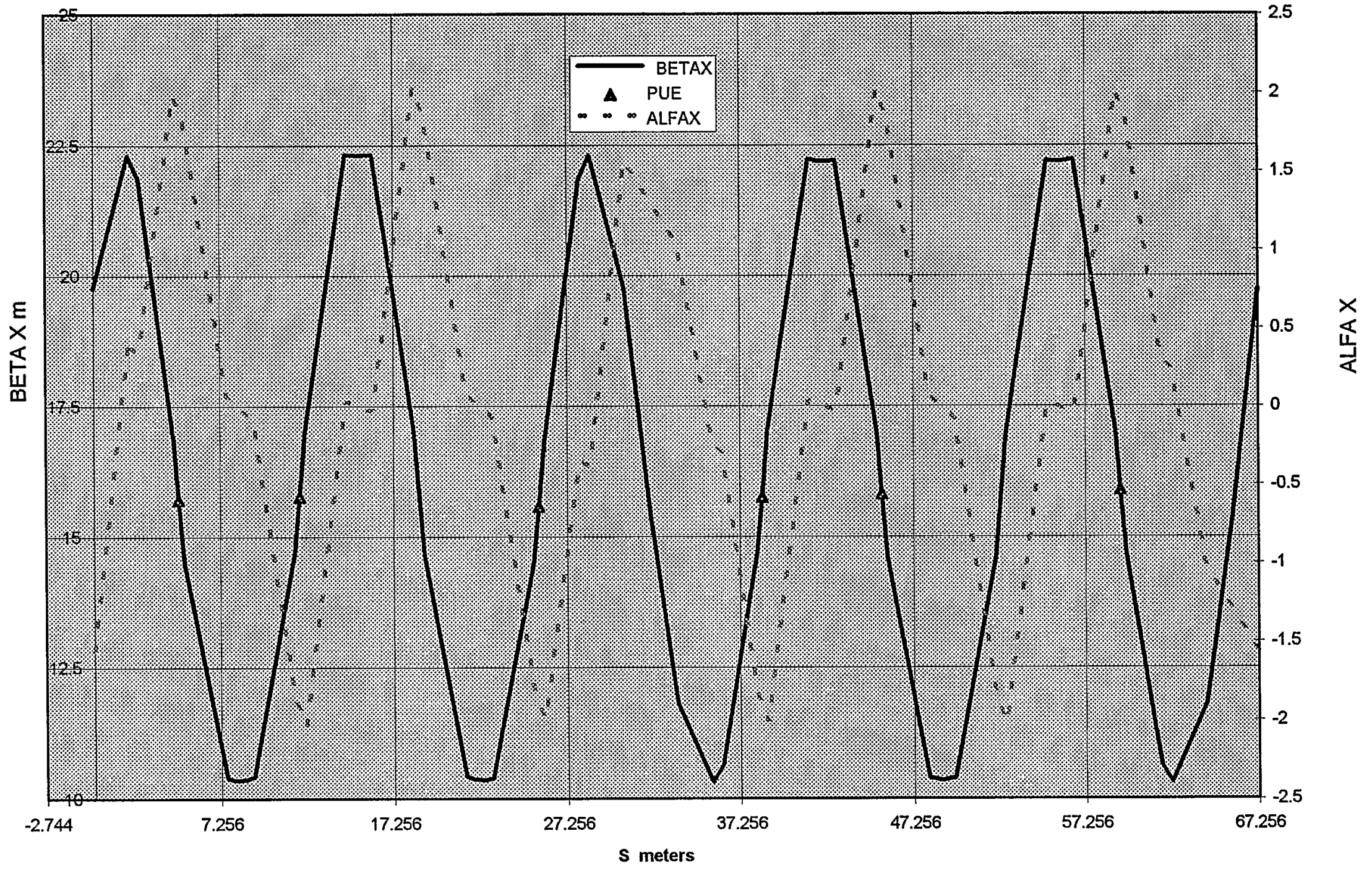


BETA Y \& ALFA Y vs S

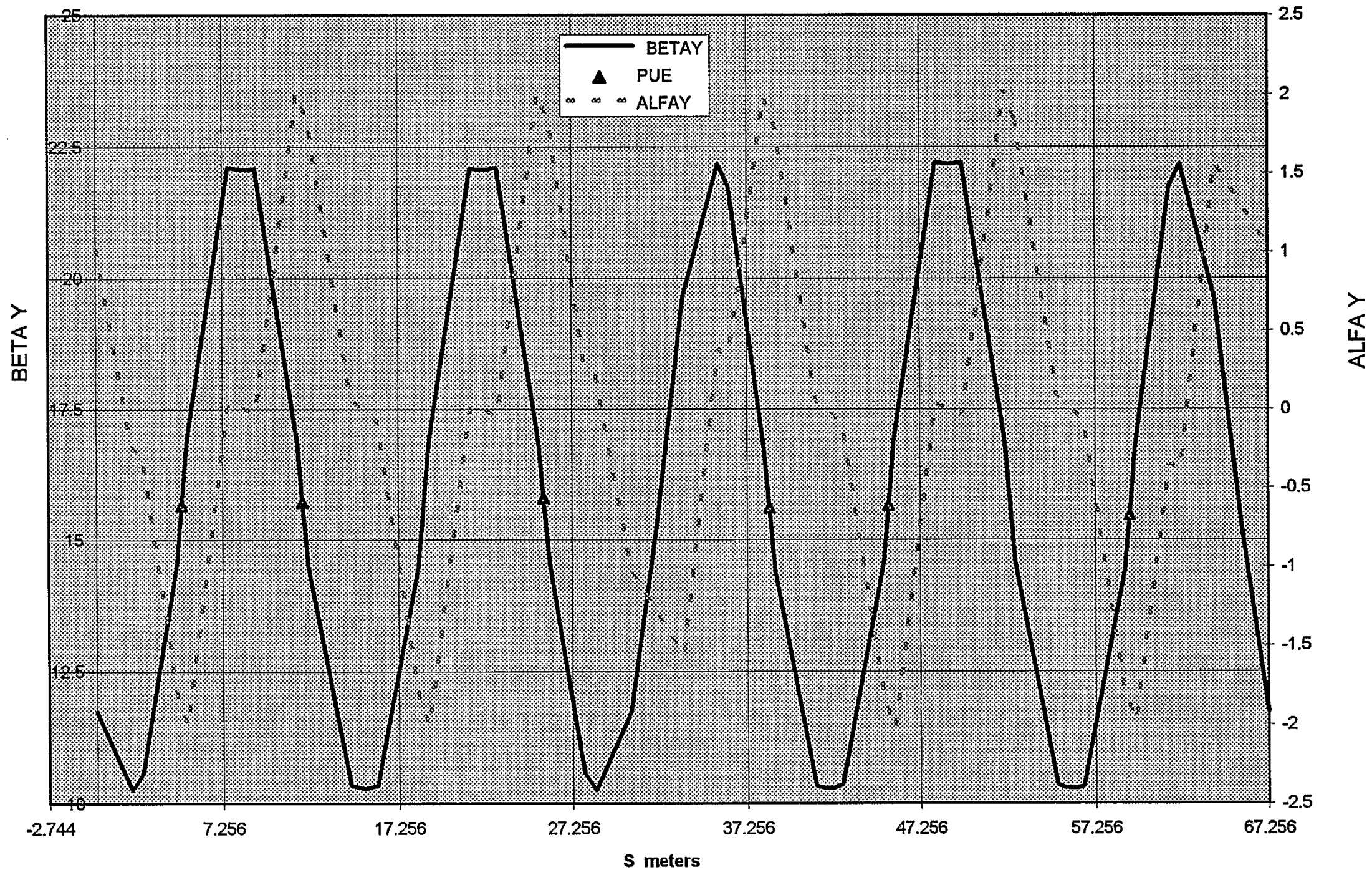


DX \& DPX vs S

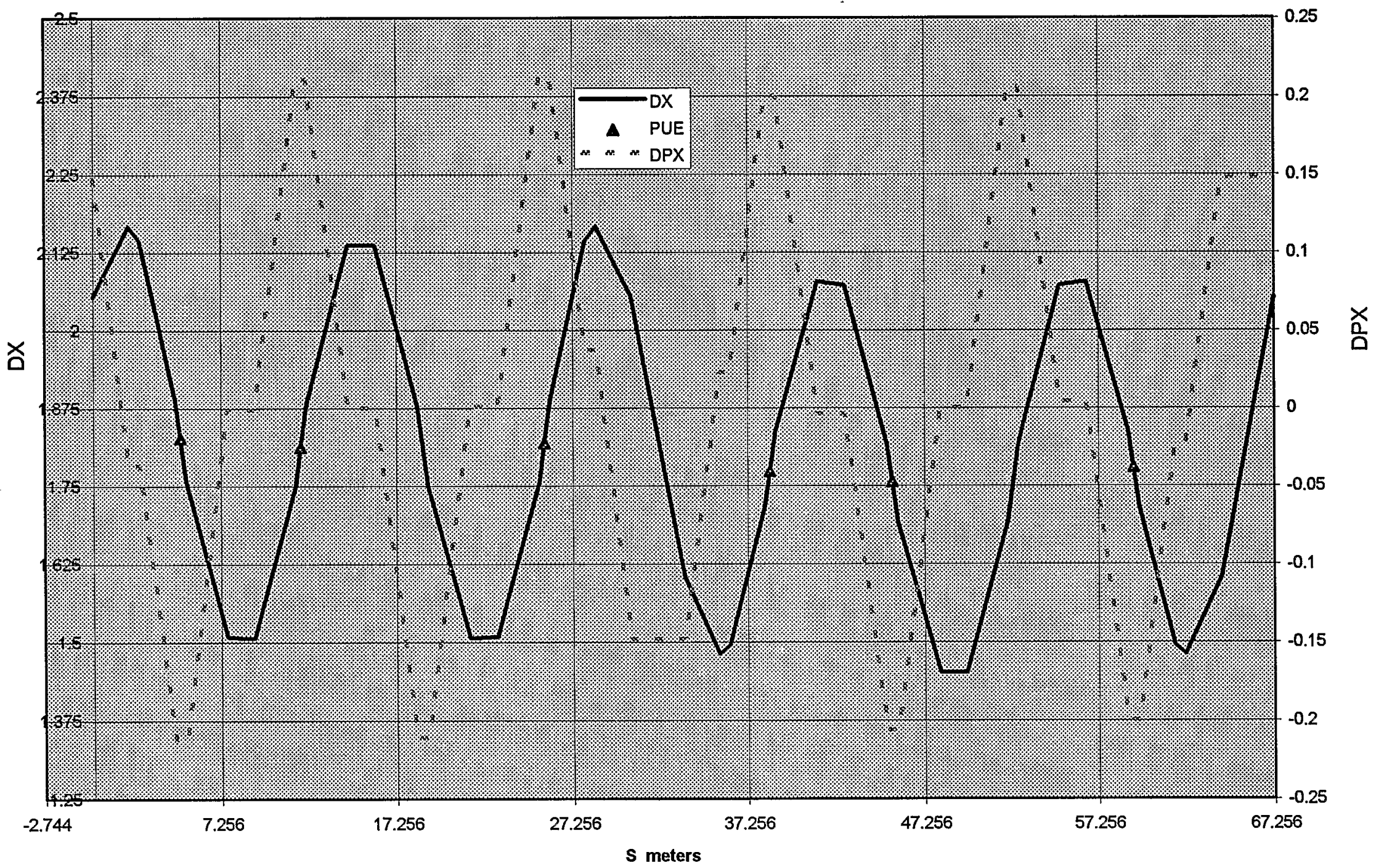




\section{Q VS P for OLD and NEW CALCULATIONS}

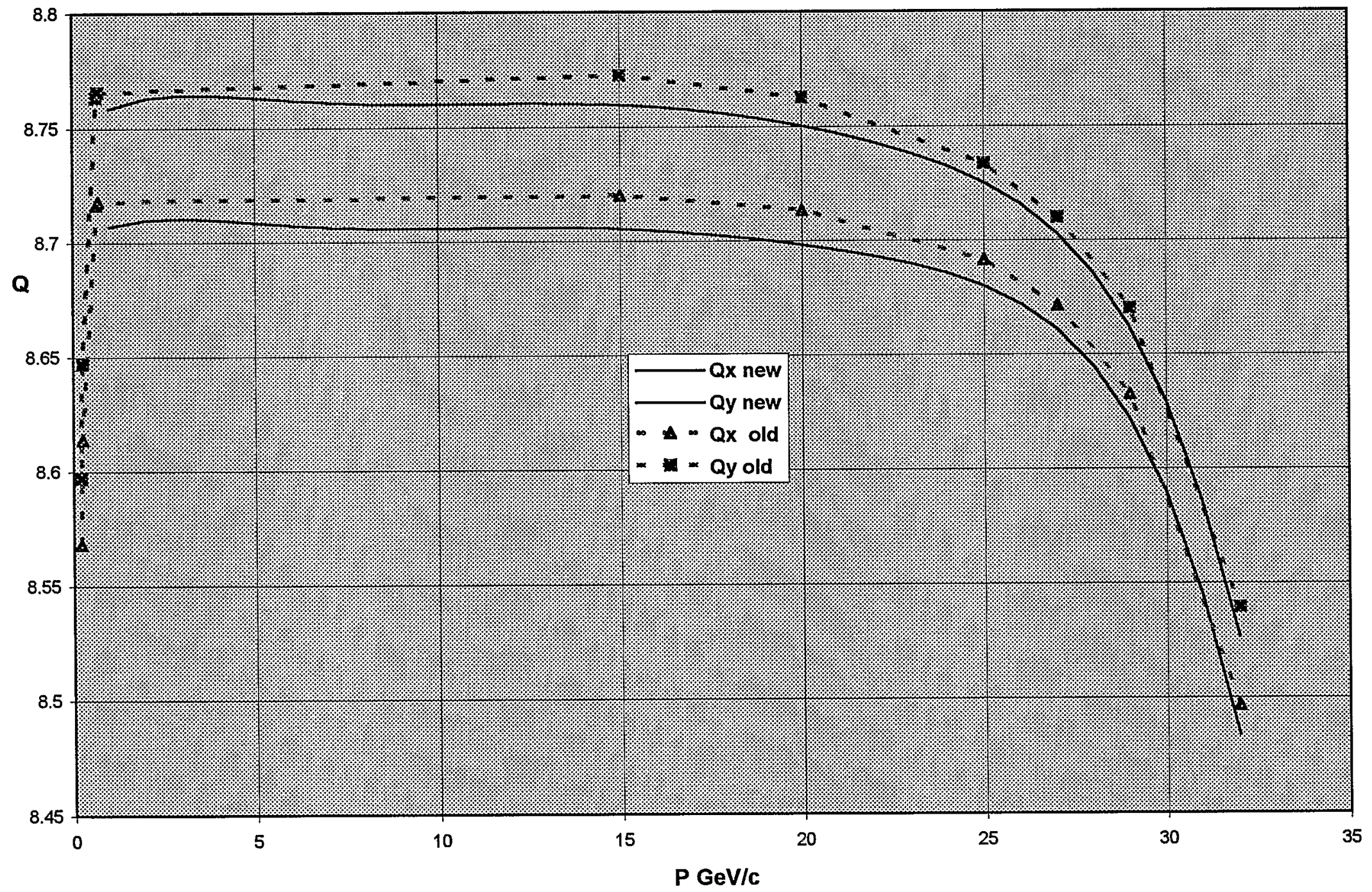

